\title{
Bed net ownership in Kenya: the impact of 3.4 million free bed nets
}

\author{
Allen Hightower*1, Rebecca Kiptui², Ayub Manya2², Adam Wolkon'1, Jodi Leigh Vanden Eng1 ', Mary Hamel1,3, \\ Abdisalan Noor 4 , Shahnaz K Sharif5, Robert Buluma6, John Vulule³, Kayla Laserson3,7, Laurence Slutsker ${ }^{1}$ and \\ Willis Akhwale?
}

\begin{abstract}
Background: In July and September 2006, 3.4 million long-lasting insecticide-treated bed nets (LLINs) were distributed free in a campaign targeting children 0-59 months old (CU5s) in the 46 districts with malaria in Kenya. A survey was conducted one month after the distribution to evaluate who received campaign LLINs, who owned insecticide-treated bed nets and other bed nets received through other channels, and how these nets were being used. The feasibility of a distribution strategy aimed at a high-risk target group to meet bed net ownership and usage targets is evaluated.

Methods: A stratified, two-stage cluster survey sampled districts and enumeration areas with probability proportional to size. Handheld computers (PDAs) with attached global positioning systems (GPS) were used to develop the sampling frame, guide interviewers back to chosen households, and collect survey data.

Results: In targeted areas, 67.5\% (95\% Cl: 64.6, 70.3\%) of all households with CU5s received campaign LLINs. Including previously owned nets, $74.4 \%$ (95\% Cl: 71.8, 77.0\%) of all households with CU5s had an ITN. Over half of CU5s (51.7\%, 95\% Cl: 48.8, 54.7\%) slept under an ITN during the previous evening. Nearly forty percent (39.1\%) of all households received a campaign net, elevating overall household ownership of ITNs to 50.7\% (95\% Cl: 48.4, 52.9\%).

Conclusions: The campaign was successful in reaching the target population, families with CU5s, the risk group most vulnerable to malaria. Targeted distribution strategies will help Kenya approach indicator targets, but will need to be combined with other strategies to achieve desired population coverage levels.
\end{abstract}

\section{Background}

The last, large-scale, group-randomized, controlled trial of insecticide-treated bed nets (ITNs) showed that ITNs were efficacious in reducing all-cause post-neonatal mortality in an area of Western Kenya with intense, perennial malaria transmission [1-3]. That trial and others [4-7] helped to define pregnant women and children 0-59 months old (CU5s) as target groups for ITNs in high transmission settings. The findings also suggested that high overall population coverage with ITNs including both target and non-target groups was critical to achieve community level protective effects [3]. These studies emphasized the beneficial effects of high ITN coverage from both health and economic perspectives.

*Correspondence: awh1@cdc.gov

1 Division of Parasitic Diseases and Malaria, Centers for Disease Control, Center for Global Health, Mailstop F22, 4770 Buford Highway, Atlanta GA 30341, USA Full list of author information is available at the end of the article
Key determinants of efficacy in this and other ITN studies were the proportion of households with ITNs (ownership), the proportion of individuals properly deploying ITNs each night (usage), and the proportion of nets properly treated with insecticide (treatment). These indicators have been adapted for programme monitoring and evaluation. Indicators for ITN ownership and usage are an integral part of a number of development goals, including those set forth in the Abuja Declaration [8], Roll Back Malaria Strategic Document (RBM) [9], and the Millennium Development Goals [10]. The 2010 RBM targets are: $80 \%$ of CU5s sleeping under an ITN during the previous night, and $80 \%$ of pregnant women sleeping under an ITN during the previous night. In the past few years, long-lasting ITNs (LLINs) have become widely available; these are nets that are treated during the manufacturing process and are protective for an estimated three years. 
One method to rapidly achieve high coverage is a largescale integrated campaign, where ITN distribution is linked to other child health interventions, such as immunizations or Vitamin A supplementation [11,12]. One of the largest of these campaigns, involving 3.4 million LLINs, was conducted in Kenya in 2006. The outcomes of this campaign, retention of campaign LLINs, and estimates of the indicators to assess Kenya's progress towards goals for ITN coverage at the household level and highrisk populations are reported here. Additionally, the survey provided a measure of the progress of ongoing and substantial efforts to distribute subsidized, socially marketed bed nets over the past four years in Kenya. The survey collected data using a novel approach that has been tested in other countries as being both statistically valid and rapid [13-16].

\section{Methods}

\section{The campaign to distribute LLINs}

1.4 million LLINs were distributed from July 8 to 14 , 2006, primarily in Nyanza and Western provinces, in association with a measles vaccination campaign. Western and Nyanza provinces were chosen to receive nets first because of a high burden of malaria-related morbidity and mortality. The remaining 2.0 million nets were distributed September 23 to 24, 2006 in malaria-affected districts in Rift Valley, Central, Eastern, and Coast Provinces. The target population to receive the nets was children under five years of age. Batches of LLINs were sent to numerous distribution centers (health facilities, schools, etc.) where caretakers would receive one net for each CU5 that accompanied them. At some centers, the children received other health interventions appropriate for the locale (measles vaccination, deworming medication, vitamin $\mathrm{A}$, and iron tablets).

\section{Survey design}

There are 46 Districts in Kenya where malaria transmission occurs. Twenty of these Districts were randomly chosen with probability proportional to size within the following three malaria transmission zones that formed the sampling strata for the survey: areas of endemic malaria - 10 districts from Nyanza, Western, and Coast provinces (2006 estimated population: 11.9 million); epidemic malaria - five districts from the Rift Valley (2006 estimated population: 8.6 million); highland/seasonal malaria - five districts from Eastern and Central Provinces (2006 estimated population: 9.2 million). The region containing Nyanza, Western, and Coast Provinces had higher sampling fractions because they contained all but one of the endemic districts in the country and are where the majority of malaria-related morbidity and mortality occurs.
Within each district, five enumeration areas were selected with probability proportional to size. A sample of 21 households was selected for each enumeration area, giving a target sample of 2,100 households. The sampling frame within each cluster was developed through the use of global positioning system (GPS) linked with hand-held computers (PDAs) to allow rapid preliminary mapping of enumeration areas, thereby allowing selection of a simple random sample of houses [13]. Sufficient numbers of districts and enumeration areas were sampled in the Nyanza and Rift Valley provinces and the other two regions to produce statistically reliable estimates and confidence intervals.

\section{Data collection}

The survey was conducted from October 17 to November 1, 2006 (Figure 1). Questions and methodology were similar to the 2003 Kenya Demographic and Health Survey (KDHS) to facilitate comparisons [17]. The survey instrument asked questions about the household, the bed nets owned by the household (if any), whether a CU5 or a woman of reproductive age had slept under each specific net during the previous evening, malaria knowledge and awareness of public media messages related to the campaign, and socio-economic questions concerning characteristics of the household. Surveyors noted whether they had actually observed the nets and if so, if they were hanging. Although pregnant women were not specifically targeted by the campaign, they were included in the survey to measure the effectiveness of the targeted campaign for CU5s in reaching them. Socioeconomic data used to construct a wealth index employing the same definitions used in the $2003 \mathrm{KDHS}$ [17].

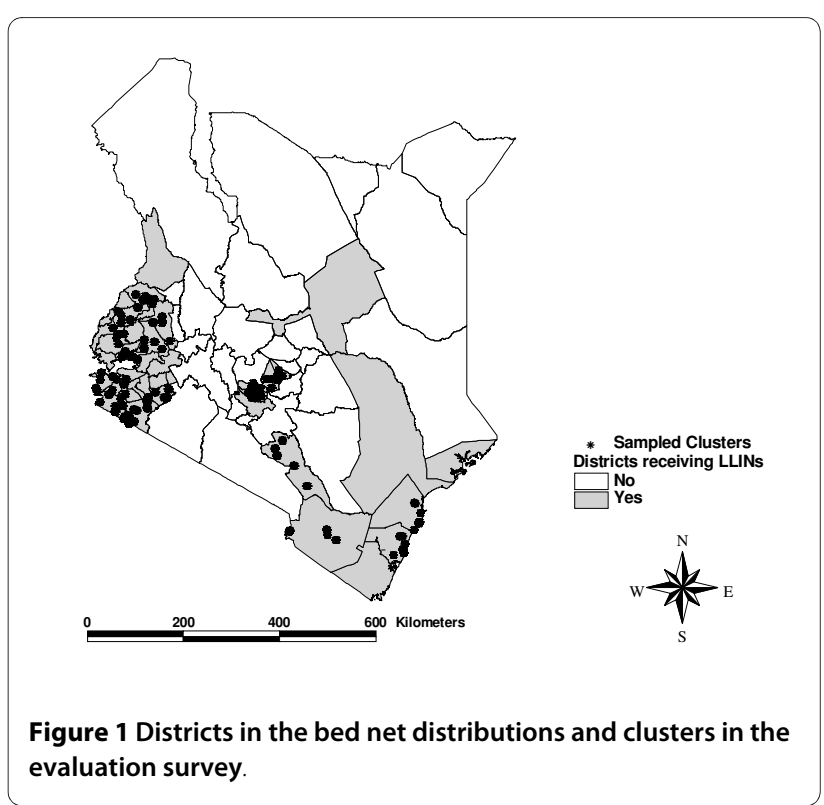


PDAs with GPS were used for data collection. The questionnaire was constructed and programmed into the PDAs with appropriate skip patterns and data checks. After a one-week training period, 11 teams of four persons each collected the data in the field over a two week period. Upon survey completion, PDAs were returned to Nairobi, where data were aggregated into a desktop database.

The survey was conducted at the end of the dry season and the early stage of the short rainy season which is not the peak time of malaria transmission. As a result it was not expected to provide the maximum measure of net usage. The objective of this survey was to assess ownership and retention of bed nets distributed during the campaign and to assess bed net ownership in general.

\section{Definitions}

A household was defined as everyone who shared a meal at a common gathering place, using the 2003 Kenya DHS definition [17]. An ITN was defined as any bed net that had been treated with insecticide in the last six months. LLINs were counted as ITNs. At the time of the survey, there were only three types of LLINs available in Kenya: SupaNet ExtraPower (socially marketed by Population Services International), Permanets (available commercially and via the campaign), and Olyset nets (available commercially and via the campaign). Pre-campaign bed net ownership levels were based on ownership of noncampaign bed nets. Changes in ownership of these nets between the campaign and the survey were assumed to be negligible. Post-campaign bed net ownership included all types of bed nets. Permanet and Olyset campaign nets had distinctive labeling and packaging. To aid identification, the PDAs used for survey data collection had pictures of each net, and its packaging and labeling, integrated into the computerized questionnaire. Equity ratio was defined as the ratio of intervention coverage proportions in the poorest quintile to the coverage in the wealthiest quintile.

\section{Statistical methods}

Consistent with the sampling design, the SAS survey procedures (SAS v9.1.3, SurveyFreq, SurveyLogistic, and SurveyMeans) were used to produce estimates and standard errors using the sampling weights that accounted for the sampling design and the different sampling fractions for each transmission zone. Estimates are precise (halfwidth of $95 \%$ confidence interval) to $2.5 \%$ on a national basis (i.e., for all areas with malaria in Kenya), $2.5-4.0 \%$ on a regional basis (the three sampling strata based on provinces grouped by the nature of their malaria endemicity), $5 \%$ for each wealth quintile, and 6\% for CU5. The survey was not designed for making precise statements on bed net ownership or usage for pregnant women. However, precision for women of reproductive age was $5 \%$ on a national basis.

Comparisons of indicators before and after the campaign were done by computing the difference in the indicators and computing 95\% confidence intervals that accounted for the survey design. The Rao Scott Chi square statistic was used for p-values. Projected numbers of various types of bed nets (ITNs, campaign nets) were computed by multiplying the number of a particular type of bed net in the survey household by its sampling weight and summing this product over all households.

\section{Ethical considerations}

The protocol was approved by the Kenya Medical Research Institute Institutional Review Board and the Centers for Disease Control and Prevention. Informed written consent was obtained from each respondent.

\section{Results}

\section{Respondents}

From October 17 through November 1, 2006, members of 2,059 households were interviewed out of 2,100 targeted. Of the 100 enumeration areas, $85 \%$ were classified as rural and $15 \%$ as urban, mirroring the characteristics of the 46 campaign districts. On a weighted basis, households with CU5s represented $51.7 \%$ of all households in the survey population. Among sampled households, $78.0 \%$ had a woman of reproductive age; of these, $37.1 \%$ did not have a CU5 (95\% CI: 34.7, 39.6\%). An estimated $8.3 \%$ of the women in the population reported themselves to be pregnant.

\section{Before vs. after campaign comparisons for key indicators} The survey estimated that 3.63 million LLINs were distributed (95\% CI: 3.39, 3.87 million), of which 3.38 million were distributed to CU5s (93.9\%) (Table 1). The campaign increased the number of bed nets owned by $50 \%$, from 7.2 to 10.9 million. Households owning only campaign nets were $13.8 \%$ of all households, which was over one-third of all households receiving campaign nets. Pre-campaign ITN household ownership could be estimated as $36.9 \%=50.7$ (post-campaign ITN/LLIN HH ownership) $-13.8 \%$ (households that only owned campaign nets in the survey), and similarly pre-campaign household ownership of any bed net could be estimated at $53.1 \%(66.9-13.8 \%)$. Prior to the campaign, socially marketed nets represented $68.7 \%$ of all nets owned. This represents approximately 5.0 million nets $(7.24$ million* 0.687). After the campaign, they represented $46.0 \%$ of all nets owned. Figure 2 presents the distribution of bed net brand ownership before and after the campaign.

The campaign increased ITN ownership for $\mathrm{HH}$ with CU5s by $28.7 \%$ (from $46.7 \%$ to $74.4 \%$, p < .0001). It 
Table 1: Key indicators, before and after the campaign *

\begin{tabular}{lll}
\hline & $\begin{array}{l}\text { Before campaign, (95\% Confidence } \\
\text { Interval) }\end{array}$ & $\begin{array}{l}\text { After campaign, (95\% Confidence } \\
\text { Interval) }\end{array}$ \\
\hline $\begin{array}{l}\text { Total Number of Campaign Bed nets } \\
\text { Distributed }\end{array}$ & none & 3.63 million, (3.39, 3.87 million) \\
\hline $\begin{array}{l}\text { Total Bed nets Owned } \\
\text { Percent of All Bed nets that were Socially }\end{array}$ & 7.24 million, $(7.14,7.34$ million) & 10.87 million, $(10.53,11.21$ million) \\
\hline $\begin{array}{l}\text { Marketed } \\
\text { HH with CU5 and ITN/LLIN }\end{array}$ & & $45.7 \%,(43.5,47.9)$ \\
\hline
\end{tabular}

* Number of bed nets of all types in survey $=3141$.

Number of ITNs (including LLINs) in survey $=2091$, found in $1080 \mathrm{HH}$.

Number of HHs in survey $=2059$

increased ITN ownership for HH with women of reproductive age by $18.6 \%$ (from $40.3 \%$ to $58.9 \%$ ).

Prior to the campaign, the percent of all nets in households with CU5s that were ITN increased with the wealth quintile. However, even in the wealthiest quintile, less than a third of the homes owned ITNs. After the campaign, the relationship between ITN ownership and wealth disappeared. The smallest increase in ITN ownership was in the wealthiest quintile, $25.5 \%$. The other wealth quintiles had increases of nearly $40 \%$ or more. The

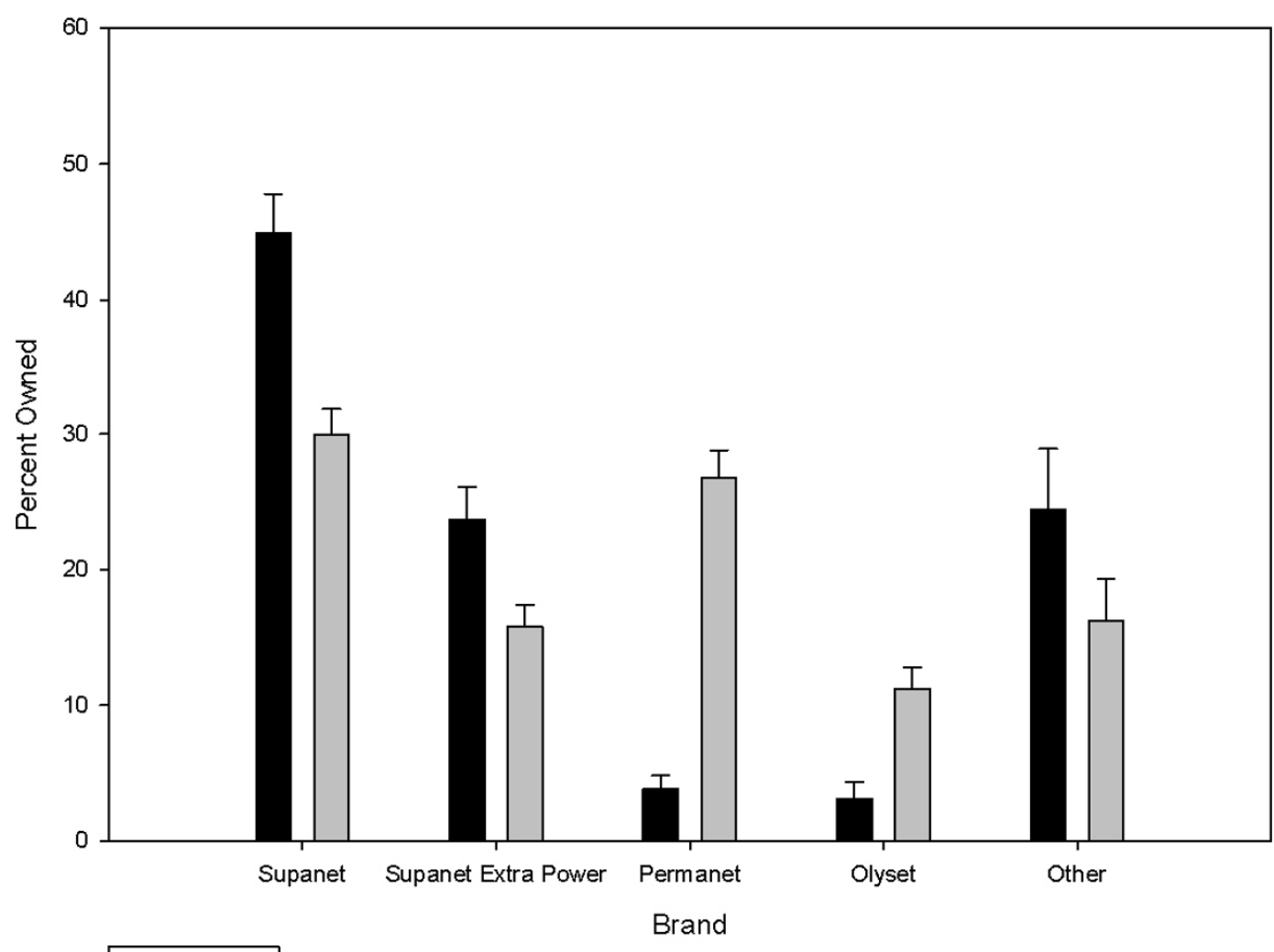

Before

After

Figure 2 Distribution of bed net brands owned before and after the campaign* * Pre-campaign: Number of bed nets $=2,105$, Post-Campaign: Number of bed nets $=3,141$. 
increases in all wealth quintiles were statistically significant $(\mathrm{p}<.001$ for all quintiles, Table 2$)$.

\section{Equity ratios}

The equity ratio for all households receiving campaign bed nets was 1.59 (95\% CI: 1.29, 1.89). This means that the poorest households were $59 \%$ more likely to receive campaign bed nets than the wealthiest households. The equity ratio for CU5s, women of reproductive age, and pregnant women sleeping under an ITN the previous night were all less than 0.8 . This means that despite the campaign, the target populations in the wealthiest quintiles were all $20 \%$ or more likely to be sleeping under an ITN. Equity ratios with $95 \%$ confidence intervals are presented in Figure 3.

\section{Bed nets}

There were 3141 bed nets in the 2059 households in the survey. Over half of households surveyed $(n=1080)$ owned ITNs. One third of all nets were campaign nets. During the survey, $70 \%$ of all nets were seen by the interviewers. Summary data with $95 \%$ confidence intervals are presented in Table 3.

Among all bed nets, $66.2 \%$ were either treated in the last six months (ie, were ITNs) or were LLINs. More than half (54.8\%) of all nets were LLINs. Among all bed nets, $43.1 \%$ were obtained at no cost. Nearly two-thirds $(67.1 \%)$ of all nets were hanging. About a third (30.6\%) of all nets were obtained at health facilities (other than as a part of the campaign), and $24.8 \%$ were obtained from dukas (small stores), hawkers, kiosks, or textile shops. Almost ninety percent $(88.4 \%)$ of nets were classified as being in good condition (no holes, tears). For the most popular brand of socially marketed bed net (a non-LLIN), 30.6\% had been treated in the last six months.

\section{Households}

About two-thirds (66.9\%) of all households had at least one bed net of any kind. Over forty percent $(41.9 \%)$ of households had more than one bed net. Over half (50.7\%) of households owned an ITN, and 39.1\% of all households had received a campaign LLIN. ITNs were hanging in $41.9 \%$ of all households. In households owning ITNs, $82.6 \%$ had at least one ITN hanging. Households that owned only campaign nets were $13.8 \%$ of all households, which was over one-third of all households receiving campaign nets. Summary data with $95 \%$ confidence intervals are presented in Table 4.

Households that had neither a CU5 nor a woman of reproductive age comprised $19.2 \%$ of all households. In this group, bed net ownership was $16.2 \%$ for any ITN, and $33 \%$ for any type of bed net. Urban households were more likely than their rural counterparts to have nets of any type. (ITNs: Odds Ratio: 1.37, 95\% CI: 1.06, 1.77; Any Net: Odds Ratio: 2.35, 95\% CI: 1.70, 2.35). Among all households that received campaign nets, $94.8 \%$ had retained all nets and $97.9 \%$ percent had retained at least one campaign net. There was no significant variation in retention by malaria transmission zone status.

\section{Children under five}

There were 1,727 CU5s living in 1113 households in the survey. Approximately two-thirds $(67.5 \%)$ of households with CU5s, received campaign LLINs. The percent of these households receiving campaign LLINs did not vary significantly by region. Just under ninety percent (87.2\%) of households with CU5s had a bed net of any type, with $74.4 \%$ owning an ITN. Summary data with $95 \%$ confidence intervals for households with CU5s are presented in Table 5.

Just over half (51.7\%) of CU5s (95\% CI: 48.8, 54.7\%) slept under an ITN during the previous night. This proportion did not vary significantly by age or sex. There was significant variation by malaria zone/geographic region: endemic: 50.3\%; epidemic: $41.4 \%$, highland/seasonal $67.9 \%(\mathrm{p}<.0001)$. In households with CU5s owning ITNs, $69.0 \%$ of children slept under them the previous night, and in households with CU5s owning ITNs that were hanging, $83.5 \%$ of children slept under them the previous night. Having received a campaign bed net greatly

Table 2: Percent of all bed nets in households with children under 5 that were ITNs (including LLINs) by wealth quintile before and after the campaign.*

\begin{tabular}{|c|c|c|c|}
\hline & $\begin{array}{l}\text { Before campaign, (95\% } \\
\text { Confidence Interval) }\end{array}$ & $\begin{array}{l}\text { After campaign, (95\% } \\
\text { Confidence Interval) }\end{array}$ & $\begin{array}{l}\text { Change ( } 95 \% \text { Confidence } \\
\text { Interval) }\end{array}$ \\
\hline Poorest & $19.9 \%,(16.0,23.9)$ & $59.1 \%,(53.7,64.5)$ & $39.2 \%,(34.2,44.2)$ \\
\hline Second Poorest & $21.4 \%,(17.3,25.5)$ & $76.2 \%,(71.5,80.9)$ & $54.8 \%,(49.6,60.0)$ \\
\hline Mid Quintile & $24.7 \%,(18.8,30.7)$ & $62.9 \%,(55.8,70.1)$ & $38.2 \%(31.4,45.0)$ \\
\hline Second Richest & $27.6 \%,(22.6,32.6)$ & $66.1 \%,(60.5,71.6)$ & $38.5 \%,(33.0,44.0)$ \\
\hline Richest & $30.6 \%,(25.4,35.9)$ & $56.1 \%,(50.1,62.1)$ & $25.5 \%,(20.5,30.4)$ \\
\hline Total & $24.4 \%,(22.3,26.6)$ & $64.5 \%,(62.0,67.1)$ & $40.1 \%,(37.6,42.6)$ \\
\hline
\end{tabular}

* Number of Households with CU5s in survey $=1113$. 


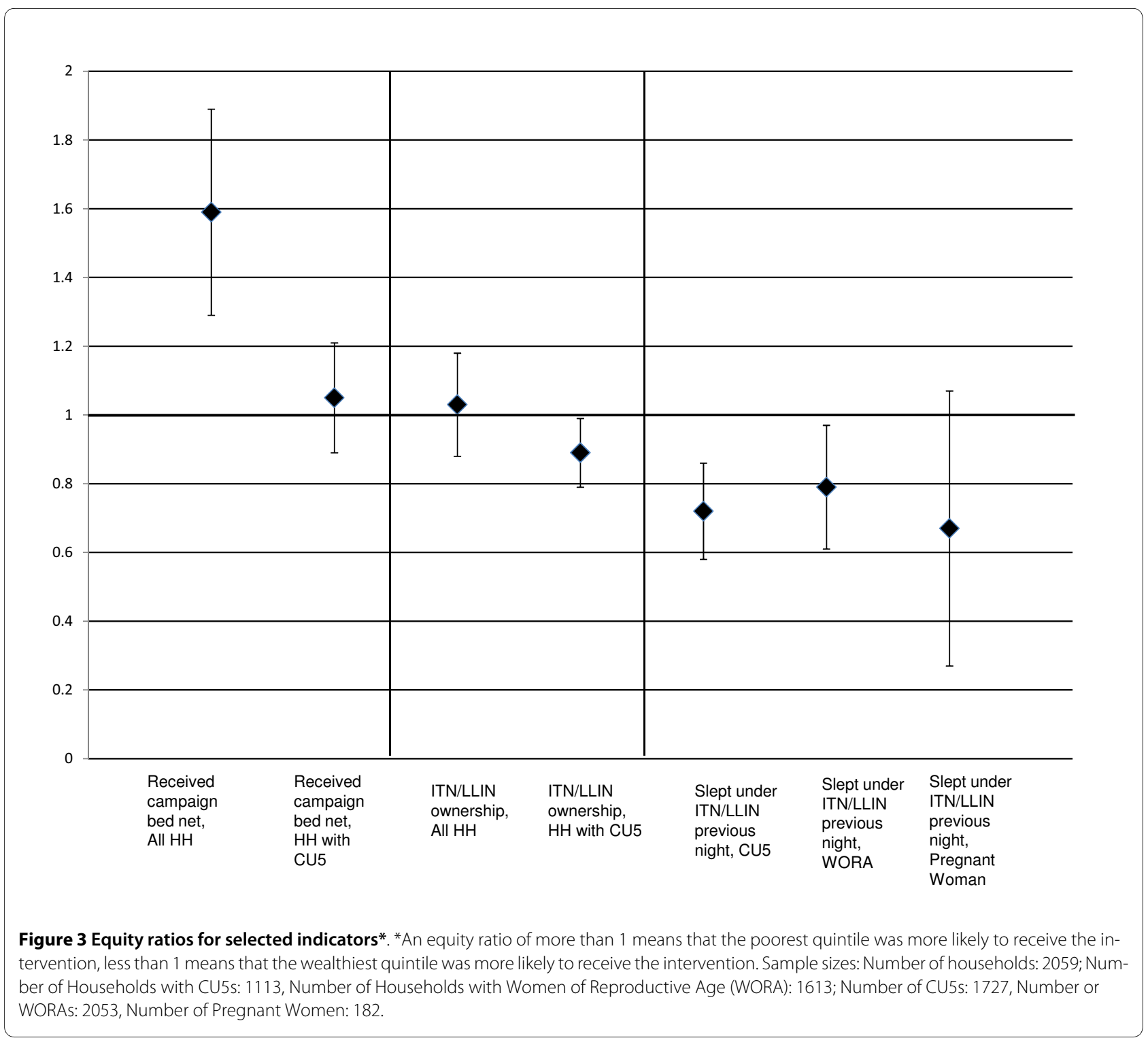

increased the likelihood that a child under 5 slept under an ITN the previous night $(67.7$ vs. $28.8 \%, \mathrm{p}<.0001)$. Overall, $56.5 \%$ of the ITNs used by CU5s were campaign nets. Summary data with $95 \%$ confidence intervals for CU5s are presented in Table 6.

\section{Women of reproductive age}

There were 2,053 women of reproductive age (15-49 years, WORAs) living in 1,613 households. There were 182 pregnant women living in 180 households in the survey. Among pregnant women, $58.4 \%$ slept under a net of any type during the previous night (95\% CI: 50.9, 65.9\%); however, only $36.3 \%$ of pregnant women slept under an ITN. (95\% CI: 29.0, 43.7\%). Among all WORAs, 33.0\% slept under an ITN during the previous night $(95 \% \mathrm{CI}$ : $30.6,35.4 \%)$. In households that owned one or more ITNs, 55.9\% of women of reproductive age slept under an
ITN during the previous night. For pregnant women, this number was $56.7 \%$. Summary data for WORAs with $95 \%$ confidence intervals are presented in Table 7 .

\section{Discussion}

The distribution of 3.4 million LLINs dramatically increased ownership, and reached two-thirds of the target households with the population most vulnerable to malaria-related morbidity and mortality. At the time of the campaign, this distribution represented the largest distribution of bed nets ever. While the distribution of bed nets in Ethiopia, undertaken later, was considerably larger (approximately 20.5 million bed nets), [18], the Kenya campaign remains the second largest.

Since 2002, Kenya had an effort to socially market subsidized bed nets [19]. This multi-year programme distrib- 
Table 3: Survey estimates related to bed nets*

\begin{tabular}{ll}
\hline & $\begin{array}{l}\text { Percent, (95\% Confidence } \\
\text { Interval) }\end{array}$ \\
\hline $\begin{array}{l}\text { Bed nets that were either an } \\
\text { ITN or LLIN }\end{array}$ & $66.2 \%,(64.7,69.1)$ \\
\hline Bed nets that were LLINs & $54.8 \%,(52.5,57.1)$ \\
\hline $\begin{array}{l}\text { Bed nets that were Campaign } \\
\text { bed nets }\end{array}$ & $33.4 \%,(31.4,35.4)$ \\
\hline $\begin{array}{l}\text { Bed nets that were Hanging } \\
\text { Bed nets in Good Condition }\end{array}$ & $67.1 \%,(65.3,69.1)$ \\
\hline $\begin{array}{l}N=3141 \text { bed nets of any type in survey } \\
N=2092 \text { ITN/LLINs in survey }\end{array}$
\end{tabular}

uted 5.0 million bed nets vs. the 3.4 million bed nets distributed as part of the campaign. Prior to the campaign, $68.7 \%$ of all bed nets were socially marketed. Afterwards, socially marketed bed nets still represented $46.0 \%$ of all bed nets owned. The vast majority of these bed nets were sold prior to the development of LLINs. A possible concern has been that the users of socially marketed bed nets might be different than the user of bed nets that are distributed free to a targeted group. Noor et al [20] notes that there is greater coverage of infants in areas where nets were received by free distributions than by social marketing.

When interpreting the impact of the campaign on bed net ownership for all households in the survey, one must remember that only $51.7 \%$ had a CU5 and were, therefore, eligible to receive a campaign LLITN. Additionally, over one third of households with women of reproductive age did not have a CU5 in the household. Households with these women, who might potentially become pregnant, were not eligible to receive campaign nets, thus not addressing the cohort of newborns that arrive following the conclusion of a campaign. A number of strategies are thus needed to achieve bed net usage and ownership targets. In addition to campaigns, other important approaches include distribution of ITNs via antenatal clinics as an efficient way to reach pregnant women. In the 2003 Kenya DHS, $88 \%$ of pregnant women received antenatal care. Therefore, increasing ITN ownership/ usage in this population towards targets via further targeted distributions is achievable.

Within the targeted population (CU5s), the campaign was highly successful. The survey found that $74.4 \%$ of households with CU5s now own an ITN, and that $87.2 \%$ own a bed net of any type. This would also represent ITN
Table 4: Survey estimates related to households $(\mathrm{HH})$ *

\begin{tabular}{ll}
\hline & $\begin{array}{l}\text { Percent, (95\% Confidence } \\
\text { Interval) }\end{array}$ \\
\hline Owns at least one bed net & $66.9 \%,(64.8,69.0)$ \\
\hline Owns > 1 bed net & $41.9 \%,(39.7,44.0)$ \\
\hline Owns an ITN/LLIN & $50.7 \%,(48.4,52.9)$ \\
\hline Owned campaign bed net & $39.1 \%,(29.9,48.1)$ \\
\hline All nets were from campaign & $13.8 \%,(12.3,15.4)$ \\
\hline Any ITN/LLIN ownership: & \\
\hline Urban HH & $57.5 \%,(51.6,63.4)$ \\
\hline Rural HH & $49.7 \%,(47.2,52.1)$ \\
\hline
\end{tabular}

Any ITN/LLIN ownership by

Wealth Quintile:

\begin{tabular}{l} 
Poorest $50.5 \%,(45.6,55.4)$ \\
\hline
\end{tabular}

Second Poorest

$60.4 \%,(55.4,65.4)$

\begin{tabular}{lc}
\hline Mid Quintile & $41.1 \%,(36.2,46.0)$ \\
\hline Second Richest & $53.6 \%,(48.5,58.7)$ \\
\hline Richest & $49.1 \%,(44.1,54.2)$
\end{tabular}

Any ITN/LLIN ownership by

malaria transmission zone:

Endemic $\quad 58.7 \%,(55.7,61.7)$

Epidemic (Rift) $\quad 48.4 \%,(44.1,52.7)$

Highlands/Seasonal 43.5\%, (39.1, 47.9)

$\mathrm{N}=2059$ households in the survey

ownership if all of these are treated (only approximately $30 \%$ were treated within the past six months at the time of the survey). However, due to logistics and costs of redipping, future net distributions will concentrate on simply replacing nets that require retreatment with LLINs. A mass distribution every three years supplemented by 
Table 5: Survey estimates for Households (HH) with Children under 5 (CU5s)*

\begin{tabular}{ll}
\hline & $\begin{array}{l}\text { Percent, (95\% Confidence } \\
\text { Interval) }\end{array}$ \\
\hline Owns at least one Bed net & $87.2 \%,(85.2,89.3)$ \\
\hline Owns an ITN/LLIN & $74.4 \%,(71.8,77.0)$ \\
\hline Owns campaign bed net & $67.5 \%,(64.6,70.3)$ \\
\hline $\begin{array}{l}\text { Any ITN/LLIN ownership by } \\
\text { Wealth Quintile of HH }\end{array}$ \\
\hline $\begin{array}{l}\text { Poorest } \\
\text { Second Poorest }\end{array}$ \\
\hline Mid Quintile & $67.2 \%,(61.1,73.2)$ \\
\hline Second Richest & $84.5 \%,(79.7,89.4)$ \\
\hline Richest & $71.9 \%,(64.4,79.5)$ \\
\hline
\end{tabular}

Owned Campaign bed nets

by Wealth Quintile

\begin{tabular}{ll}
\hline Poorest & $61.6 \%,(55.9,67.4)$ \\
\hline Second Poorest & $81.4 \%,(76.7,86.2)$ \\
\hline Mid Quintile & $61.9 \%,(54.1,69.7)$ \\
\hline Second Richest & $70.8 \%,(64.5,77.1)$ \\
\hline Richest & $58.4 \%,(51.1,65.7)$ \\
\hline
\end{tabular}

$\mathrm{N}=1113$ Households with Children Under 5.

those available through health facilities would seem to be the simplest way to keep large numbers of vulnerable populations well supplied with viable LLINs

The survey results demonstrated that retention of campaign bed nets was very high (95\% of all households retained all campaign nets). Therefore, few campaign bed nets were being resold or given away. This finding has been replicated elsewhere and has remained the case in later follow-up surveys [16].

Despite the timing of the survey, usage figures were much higher than previous surveys (51.7\% of CU5s slept
Table 6: Survey estimates for Children under 5 (CU5s)*

\begin{tabular}{ll}
\hline Variable & Percent, \\
& $(95 \%$ Confidence Interval $)$
\end{tabular}

Percent CU5s sleeping under ITN $\quad 51.7,(48.8,54.7)$ previous night

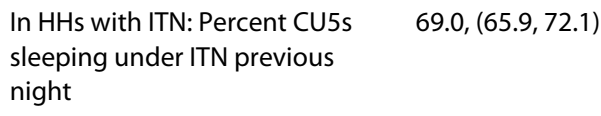

\begin{tabular}{lc}
\hline Urban & $58.6,(50.5,66.8)$ \\
\hline Rural & $51.1,(47.9,54.3)$ \\
\hline
\end{tabular}

Slept Under ITN previous night:

Malaria Region:

\begin{tabular}{lc}
\hline Endemic & $50.3,(46.3,54.3)$ \\
\hline Epidemic & $41.4,(35.7,47.1)$ \\
\hline Highland/seasonal & $67.9,(62.3,74.5)$ \\
\hline
\end{tabular}

CU5s receiving Campaign Nets by Wealth Quintile of $\mathrm{HH}$ :

\begin{tabular}{ll}
\hline Poorest & $48.6,(43.0,54.1)$ \\
\hline Second Poorest & $70.9,(65.4,76.3)$ \\
\hline Mid Quintile & $59.2,(51.4,67.0)$ \\
\hline Second Richest & $63.6,(56.8,70.3)$ \\
\hline Richest & $53.1,(45.9,60.4)$ \\
\hline
\end{tabular}

$\mathrm{N}=1727$ Children under 5 in the survey. 
Table 7: Survey estimates for Women of Reproductive Age (WORAs)*

\begin{tabular}{ll}
\hline Variable & Percent, (95\% \\
Confidence Interval)
\end{tabular}

Percent WORAs sleeping under ITN previous $33.0,(30.6,35.4)$ night

In HHs with ITN: Percent WORAs sleeping $\quad 55.9,(52.7,59.1)$
under ITN previous night

In HHs with ITN hanging: Percent WORAs $\quad 67.5,(64.2,70.8)$ sleeping under ITN previous night

WORAs in $\mathrm{HH}$ receiving campaign bed nets $\quad 46.0 \%,(43.3,48.8)$

Slept Under ITN previous night:

\begin{tabular}{lc}
\hline Urban & $47.3,(40.7,53.9)$ \\
\hline Rural & $31.2,(28.8,33.7)$ \\
\hline
\end{tabular}

Slept Under ITN previous night: Malaria Region:

\begin{tabular}{lc}
\hline Endemic & $35.5,(32.2,38.8)$ \\
\hline Epidemic & $30.1,(25.9,34.4)$ \\
\hline Highland/seasonal & $32.4,(27.6,37.2)$
\end{tabular}

WORAs Sleeping under ITN/LLIN previous night

\begin{tabular}{lc}
\hline Poorest & $28.2,(23.3,33.1)$ \\
\hline Second Poorest & $33.3,(28.1,38.4)$ \\
\hline Mid Quintile & $31.2,(25.8,36.6)$ \\
\hline Second Richest & $35.5,(30.3,40.7)$ \\
\hline Richest & $35.6,(30.1,41.0)$ \\
\hline $\mathrm{N}=2053$ Women of Reproductive age in the survey.
\end{tabular}

under an ITN vs. 4.6\% CU5s under any net the previous night in 2003) [17]. In homes with ITNs, the usage rates were even higher (69.0\%) and higher still in homes where ITNs were hanging $(83.5 \%)$ Finally, in perhaps the best measure of the impact of the campaign, CU5s in homes receiving campaign bed nets were approximately $40 \%$ more likely to sleep under an ITN during the previous night (67.7 vs. $28.8 \%$, p <.0001). Nonetheless, the survey found that a substantial number of bed nets were not being hung or used. It is notable that households in the poorest wealth quintile were $59 \%$ more likely to receive campaign bed nets, but that target populations in the wealthiest quintile were still $20 \%$ or more likely than those in the poorest quintile to actually have slept under an ITN during the previous evening (Figure 3). Behavioural change and education programmes could help close the gap between ownership and usage. These efforts will be a vital part of any strategy to meet bed net ownership and usage targets [20,21].

\section{Conclusion}

The campaign successfully increased ITN ownership and usage in targeted vulnerable populations. The survey identified the need for educational and behavioral change campaigns to promote proper usage by vulnerable populations. Finally, the survey quantified the populations of interest not covered by the targeted distribution and for some of these populations, and helped delineate obvious next steps for increasing LLINs ownership.

Future efforts should target getting bed nets to the remaining populations that still do not have them, address eventual replacement of regular ITNs with LLINs, and encourage proper use of the bed nets.

\section{Competing interests}

The authors declare that they have no competing interests.

\section{Authors' contributions}

AH drafted the protocol, helped analyse the data, and wrote the manuscript. RK assisted with the protocol and provided scientific direction for the bed net campaign and the survey. AM provided logistical support for the bed net campaign, and helped with protocol development. AW provided data collection and analysis support, JE provided data collection and analysis support, $\mathrm{MH}$ provided scientific assistance on the protocol and the manuscript. AN provided assistance in the mapping and sampling process as well as the manuscript. SS directed the survey and provided input for both the protocol and manuscript. RB provided sampling expertise and data collection assistance. JV provided scientific advice and helped write the protocol and manuscript. KL provided assistance in the protocol and manuscript writing. LS helped write the manuscript and provided assistance for the survey. WA helped write the protocol and provided logistical support for both the bed net campaign and survey.

All authors read and approved the final manuscript.

\section{Acknowledgements}

The authors are grateful to the Ministry of Health survey team for their invaluable contribution collection the data for the field survey. We thank the Ministry of Health, and the Division of Malaria Control professional staff for providing support and supervision for the survey. This paper was published with permission from the Director, KEMRI. 


\section{Author Details}

1Division of Parasitic Diseases and Malaria, Centers for Disease Control, Center for Global Health, Mailstop F22, 4770 Buford Highway, Atlanta GA 30341, USA 2Division of Malaria Control, Ministry of Public Health and Hygiene, KNH Grounds, P.O. Box 20750, Nairobi, Kenya, ${ }^{3}$ Kenya Medical Research Institute, Center for Global Health Research, Off Busia Road, Kisian, Kenya, ${ }^{4}$ Malaria Public Health and Epidemiology Group, Centre for Geographic Medicine Research-Coast, Kenya Medical Research Institute/Wellcome Trust Research Programme, P.O. Box 43640, 00100 GPO, Nairobi, Kenya, ${ }^{5}$ Ministry of Health, Office of the Director of Public Health and Sanitation, Afya House, Cathedral Road, P.O. Box 30016, Nairobi, Kenya P.O. Box 30016, Nairobi, 6Kenya National Bureau of Statistics, Herufi House, Lt. Tumbo Lane, P.O. Box 30266-00100 GPO, Nairobi, Kenya and ${ }^{7}$ Center for Global Health, Centers for Disease Control, 1600 Clifton Road, Atlanta GA 30333, USA

Received: 23 March 2010 Accepted: 24 June 2010

Published: 24 June 2010

\section{References}

1. Hawley WA, Ter Kuile FO, Steketee RS, Nahlen BL, Terlouw DJ, Gimnig JE, Shi YP, Vulule JM, Alaii JA, Hightower AW, Kolczak MS, Kariuki SK, PhillipsHoward PA: Implications of the Western Kenya permethrin-treated bed net study for policy, program implementation, and future research. Am J Trop Med Hyg 2003, 68(Suppl 4):168-173.

2. Phillips-Howard PA, Nahlen BL, Kolczak MS, Hightower AW, Ter Kuile FO, Alaii JA, Gimnig JE, Arudo J, Vulule JM, Odhacha A, Kachur SP, Schoute E, Rosen DH, Sexton JD, Oloo AJ, Hawley WA: Efficacy of permethrintreated bed nets in the prevention of mortality in young children in an area of high perennial malaria transmission in Western Kenya. Am J Trop Med Hyg 2003, 68(Suppl 4):23-29.

3. Hawley WA, Phillips-Howard PA, Ter Kuile FO, Terlouw DJ, Vulule JM, Ombok M, Nahlen BL, Gimnig JE, Kariuki SK, Kolczak MS, Hightower AW: Community-wide effects of permethrin-treated bed nets on child mortality and malaria morbidity in Western Kenya. Am J Trop Med Hyg 2003, 68(Suppl 4):121-127.

4. D'Alessandro U, Olaleye BO, McGuire W, Langerock P, Bennett S, Aikins MK, Thompson MC, Cham MK, Greenwood BM: Mortality and morbidity from malaria in Gambian children after introduction of an impregnated bednet programme. Lancet 1995, 345:479-483.

5. Binka FN, Kubaje A, Adjuik M, Williams LA, Lengeler C, Maude GH, Armah GE, Kajihara B, Adiamah JH, Smith G: Impact of permethrin-impregnated bednets on child mortality in Kassens-Nankana District Ghana: A randomized controlled trial. Trop Med Int Health 1996, 1:147-154.

6. Nevill CG, Some ES, Mung'la VO, Mutemi W, New L, Marsh K, Lengeler C, Snow RW: Insecticide treated bednets reduce mortality and severe morbidity from malaria among children on the Kenyan coast. Trop Med Int Health 1996, 1:139-146.

7. Habluetzel A, Diallo DA, Esposito F, Lamizana L, Pagnoni F, Lengeler C, Traore' C, Cousens SN: Do insecticide treated curtains reduce all-cause mortality in Burkina Faso? Trop Med Int Health 1997, 2:-862.

8. Roll Back Malaria: The Abuja Declaration and the Plan of Action (The African Summit on Roll Back Malaria Abuja, 25 April, 2000) [http:// www.rollbackmalaria.org/docs/abuja declaration.pdf]. Accessed 9 March 2010

9. Roll Back Malaria: Roll Back Malaria Global Strategic Plan 2005-2015. [http://www.rollbackmalaria.org/forumV/globalstrategicplan.htm]. (Accessed 9 March 2010)

10. United Nations Development Program: Millenium Project. Final Report to United Nations Secretary General. London/Sterling, VA: United Nations; 2005

11. Grabowsky M, Farrell N, Hawley W, Chimumbwa J, Hoyer S, Wolkon A, Selanikio J: Integrating insecticide-treated bednets into a measles vaccination campaign achieves high, rapid and equitable coverage with direct and voucher-based methods. Trop Med Int Health 2005, 10:1151-1160.

12. Grabowsky M, Farrell N, Hawley W, Chimumbwa J, Nobiya T, Ahun M, Hoyer S, Wolkon A, Selanikio J: Ghana and Zambia: achieving equity in the distribution of insecticide-treated bednets through links with measles vaccination campaigns. Reaching the poor. The World Bank; 2005.

13. Vanden Eng JL, Wolkon A, Frolov AS, Terlouw DJ, Eliades MJ, Morgah K, Takpa V, Dare A, Sodahlon YK, Doumanou Y, Hawley WA, Hightower AW:
Use of handheld computers with global positioning systems for probability sampling and data entry in household surveys. Am J Trop Med Hyg 2007, 77:393-399.

14. Eliades MJ, Wolkon A, Morgah K, Crawford SB, Dorkenoo A, Sodahlon Y, Hawley WA, Hightower AW, Ter Kuile FO, Terlouw J: Burden of malaria at community level in children less than 5 years of age in Togo. Am J Trop Med Hyg 2006, 75:622-629.

15. Kulkarni MA, Vanden Eng JL, Desrochers RE, Cotte AH, Goodson JL, Johnston A, Wolkon A, Erskine M, Berti P, Rakotoarisoa A, Ranaivo L, Peat J: Contribution of integrated campaign distribution of long-lasting insecticidal nets to coverage of target groups and total populations in malaria-endemic areas in Madagascar. Am J Trop Med Hyg 2010, 82:420-425.

16. Thwing J, Hochberg N, Vanden Eng JL, Issifi S, Eliades MJ, Minkoulou E, Wolkon A, Gado H, Ibrahim O, Newman RD, Lama M: Insecticide-treated net ownership and usage in Niger after a nationwide integrated campaign. Trop Med Int Health 2008, 13:1-8.

17. Central Bureau of Statistics, (CBS) [Kenya], Ministry of Health [Kenya], and ORC Macro: Kenya Demographic and Health Survey 2003. Calverton, Maryland: $\mathrm{CBS}, \mathrm{MOH}$, and ORC Macro; 2004

18. One.org: HIV/AIDS: Tuberculosis and Malaria in sub-Saharan Africa: Ethiopia: bed nets reduce child deaths from malaria. [http:// www.one.org/c/us/progressreport/778/]. Accessed 9 March 2010

19. Tilson D: The Social Marketing of Insecticide-Treated Nets (ITNs) in Kenya. [http://www.gwumc.edu/sphhs/departments/pch/phcm/ casesjournal/volume1/commissioned/cases 1 12.pdf]. Accessed 9 March 2010

20. Noor AM, Mutheu JJ, Tatem AJ, Hay SI, Snow RI: Insecticide-treated net coverage in Africa: mapping progress in 2000-07. Lancet 2009, 373:58-67.

21. Noor AM, Amin AA, Akwhale WS, Snow RW: Increasing access and decreasing inequity to insecticide-treated net use among rural Kenyan children. PLoS Medicine 2007, 4:e255.

doi: 10.1186/1475-2875-9-183

Cite this article as: Hightower et al., Bed net ownership in Kenya: the impact of 3.4 million free bed nets Malaria Journal 2010, 9:183

\section{Submit your next manuscript to BioMed Central and take full advantage of:}

- Convenient online submission

- Thorough peer review

- No space constraints or color figure charges

- Immediate publication on acceptance

- Inclusion in PubMed, CAS, Scopus and Google Scholar

- Research which is freely available for redistribution 